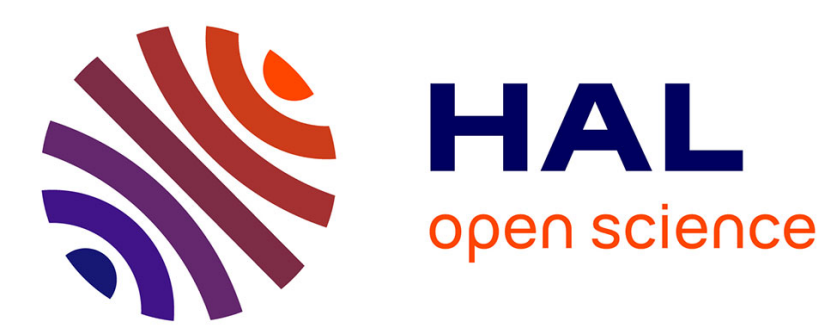

\title{
Réalisation et étude d'un spectromètre de masse radiofréquence
}

J. Peresse, G. Sinou, F. Tuffin

\section{To cite this version:}

J. Peresse, G. Sinou, F. Tuffin. Réalisation et étude d'un spectromètre de masse radiofréquence. Revue de Physique Appliquée, 1966, 1 (4), pp.257-261. 10.1051/rphysap:0196600104025700 . jpa-00242730

\section{HAL Id: jpa-00242730 https://hal.science/jpa-00242730}

Submitted on 1 Jan 1966

HAL is a multi-disciplinary open access archive for the deposit and dissemination of scientific research documents, whether they are published or not. The documents may come from teaching and research institutions in France or abroad, or from public or private research centers.
L'archive ouverte pluridisciplinaire HAL, est destinée au dépôt et à la diffusion de documents scientifiques de niveau recherche, publiés ou non, émanant des établissements d'enseignement et de recherche français ou étrangers, des laboratoires publics ou privés. 


\title{
REVUE DE PHYSIQUE APPLIQUÉE
}

\author{
Supplément au Journal de Physique
}

\section{RÉALISATION ET ÉTUDE D'UN SPECTROMÈTRE DE MASSE RADIOFRÉQUENCE}

\author{
Par J. PERESSE, G. SINOU et F. TUFFIN, \\ Faculté des Sciences, Brest.
}

\begin{abstract}
Résumé. - Nous avons réalisé trois spectromètres de masse radiofréquence comportant respectivement 8,16 et 24 étages. Nous étudions particulièrement celui de 24 étages ; les usages possibles des 8 et 16 étages sont indiqués.
\end{abstract}

Abstract. - We have constructed three radio frequency mass spectrometers (respectively 8,16 and 24 stages). We have studied more particularly the 24 stage one ; possible uses of 8 and 16 stages are indicated.

Parmi les différents spectromètres de masse non magnétiques, ceux du type radiofréquence ont l'avantage d'être peu encombrants, ce qui permet de les intégrer aisément dans des montages destinés à des études d'ionisation.

Ces appareils sont constitués, soit de grilles [1], $[2],[3]$, soit de disques percés de trous circulaires [5], [6].

Les systèmes comportant des disques ne permettent pas d'obtenir des courants ioniques intenses. En effet, chaque disque ne peut être assimilé à une équipotentielle plane que si l'orifice est de petit diamètre ; dans ce cas, l'intensité du faisceau détectable est faible.

Les dispositifs à grilles peuvent donner des courants ioniques plus importants. Aussi avons nous préféré utiliser des grilles pour la réalisation de nos trois spectromètres radiofréquence comportant respectivement 8,16 et 24 étages.

Principe. - Les ions créés dans la chambre de collision par bombardement électronique ont une vitesse $v$ lorsqu'ils atteignent la première grille du spectromètre de masse (fig. 1).

Cette vitesse dépend du rapport $q / m$ et de la différence de potentiel $V_{\text {a }}$ entre la chambre et la grille accélératrice. Entre deux grilles consécutives est appliquée une tension alternative :

$$
V_{\mathrm{m}} \sin (\omega t+\theta) \text {. }
$$

Les ions reçoivent une énergie $\delta W$ de la part du champ radiofréquence et le gain d'énergie varie selon le rapport $q / m$ de l'ion incident. En effet, ce gain est maximum $\left(\delta W_{\mathrm{m}}\right)$ pour un ion traversant l'espace entre les deux grilles pendant une demipériode et atteignant la première grille lorsque l'angle de phase du champ radiofréquence est $\theta=\pi$.

Pour les ions dont la vitesse est différente (rapport $q / m$ différent), l'accroissement d'énergie est moindre.

Seuls les ions ayant reçu l'énergie maximum pourront donc franchir une harrière de potentiel $V_{\boldsymbol{r}}$ telle que $V_{\mathrm{a}}<V_{\mathrm{r}}<V_{\mathrm{a}}+\delta W$ les autres étant arrêtés.

Théorie. - La théorie de ces spectromètres de masse radiofréquence a été élaborée par Redhead [3] et par Redhead et Growell [4]. Un bref résumé de cette théorie est présenté ci-dessous en faisant les approximations suivantes :

10 L’énergie gagnée par les ions dans le champ radiofréquence est supposée négligeable par rapport à l'énergie due à l'accélération initiale $V_{\mathrm{a}}$.

$2^{\text {n }}$ Toutes les grilles sont supposées agir comme des équipotentielles planes parfaites.

Soit $\theta$ l'angle de phase tel que $V_{\mathrm{m}} \sin \theta$ soit la tension alternative lorsque l'ion arrive sur la première grille, $V_{\mathrm{m}}$ étant la valeur maximum de cette tension. Soit $f$ la fréquence, $V_{\mathrm{a}}$ le potentiel accélérateur initial du faisceau ionique, $v$ et $m$ respectivement la vitesse et la masse de l'ion. Étant donné les hypothèses faites précédemment, le gain d'énergie sera [1]:

$$
\delta W=\Delta\left(\frac{1}{2} m v^{2}\right)=v \cdot \Delta(m v)=v \int F \cdot \mathrm{d} t,
$$


où $F$ est la force due au champ RF appliquée à l'ion.

Le champ. électrique entre la $n^{\text {lème }}$ et la $(n+1)^{\text {rème }}$ grille est

$$
(-1)^{n}\left(V_{m} / s\right) \sin (\omega t+\theta)
$$

Si l'on a $N$ étages l'énergie gagnée par l'ion lors de sa traversée du système est :

$\delta W=V_{\mathrm{m}} \frac{q v}{s} \sum_{n=1}^{N}(-1)^{n+1}$

$$
\int_{(n-1) s / v}^{n s / v} \sin (\omega t+\theta) \mathrm{d} t
$$

où $s$ est la distance séparant deux grilles. Si l'angle de transit pour un étage est défini par :

$$
\alpha=\frac{s \omega}{v}=s \omega\left(\frac{m}{2 q V_{\mathrm{a}}}\right)^{1 / 2}
$$

On obtient :

$\delta W=\frac{q V_{\mathrm{m}}}{\alpha} \sum_{n=1}^{N}(-1)^{n+1} \int_{(n+1) \alpha / \omega}^{n \alpha / \omega} \sin (\omega t+\theta) \mathrm{d} t$

soit après intégration :

$\delta W=\frac{q V_{\mathrm{m}}}{\alpha} \sum_{n=1}^{N}(-1)^{n+1}[-\cos (\omega t+\theta)]_{(n-1) \alpha / \omega}^{n \alpha / \omega}$

Si $N$ est pair on peut mettre l'expression ci-dessus sous la forme :

$$
\begin{aligned}
\delta W=\frac{q V_{\mathrm{m}}}{\alpha}\left\{2 \sum_{n=0}^{N}(-1)^{n+1}[-\cos (n \alpha+\theta)]\right. \\
-[\cos \theta+\cos (N \alpha+\theta)]\}
\end{aligned}
$$

ou encore :

$$
\begin{aligned}
\delta W=\frac{q V_{\mathrm{m}}}{\alpha}\left\{2 \sum_{n=0}^{N} \cos (\right. & n \alpha+\theta) \\
& -4 \sum_{n=0}^{N / 2-1} \cos (2 n \alpha+\alpha+\theta) \\
- & {[\cos \theta+\cos (N \alpha+\theta)]\} . }
\end{aligned}
$$

et l'on obtient finalement :

$$
\delta W=\frac{-2 q V_{\mathrm{m}}}{\alpha} \cos \left(\frac{N \alpha}{2}+\theta\right) \operatorname{tg} \frac{\alpha}{2} \sin \frac{N \alpha}{2} .
$$

Lorsque :

$$
\frac{N \alpha}{2}+\theta=p \pi \quad(p \text { entier })
$$

l'énergie gagnée $(\cos p \pi=+1)$ ou perdue $(\cos p \pi=-1)$ est maximum.

Cette énergie maximum étant :

$$
\delta W_{\mathrm{m}}=\frac{2 q V_{\mathrm{m}}}{\alpha}\left|\operatorname{tg} \frac{\alpha}{2} \sin \frac{N \alpha}{2}\right| .
$$

La plus grande valeur de $W_{\mathrm{m}}$ est obtenue pour un angle de transit voisin de $\pi(3)$ soit :

$$
\delta W_{\mathrm{m}}(\pi)=2 N q V_{\mathrm{m}} / \pi
$$

ce qui donne une valeur théorique pour $N=24$ et $V_{\mathrm{m}}=6$ volts :

$$
W_{\mathrm{m}}=91 \mathrm{~V} \text {. }
$$

Pouvoir de résolution. - Le pouvoir de résolution [3] est défini par :

$$
R=\frac{M_{1}-M_{2}}{M_{0}} \times 100
$$

$M_{0}$ étant la masse de l'ion " résonnant " $M_{1}$ et $M_{2}$ étant parmi les masses des ions repoussés par la retardatrice celles qui sont les plus voisines de $M_{0}$.

On obtient alors

$R=\frac{(\pi-\delta)^{2}-(\pi+\delta)^{2}}{\pi^{2}} \times 100=\frac{-4 \delta}{\pi} \times 100$

avec

$$
\delta^{2}=3 k \pi / N\left(N^{2}-1\right)
$$

et $k=\delta V / V$ où $\delta V$ est défini par l'équation

ce qui donne

$$
q . \delta V=\delta W
$$

soit

$$
\begin{aligned}
& R^{2}=48 \times 10^{4} k / N\left(N^{2}-1\right) \pi \\
& R=10^{2} \sqrt{48 k / N\left(N^{2}-1\right) \pi} \% .
\end{aligned}
$$

Par conséquent, dans les mêmes conditions d'emploi que Redhead, c'est-à-dire avec $K=1 / 12$, nous aurions $R=0,96 \%$ au lieu de $R=1,26 \%$.

Description de l'appareil. - La figure 1 donne le schéma de principe de nos trois spectromètres et la photographie présente un des appareils réalisés.

a) Production du faisceau d'ions. - Les ions sont créés par bombardement électronique. Les électrons émis par un filament sont accélérés vers la chambre de collisions.

Pour éviter le plus possible la pénétration des lignes de force dans la chambre de collisions, nous avons disposé à la sortie de cette dernière une grille peu transparente $(80 \%)$; ceci diminue en outre la dispersion en énergie des ions issus de la chambre. Pour diminuer la divergence du faisceau entre la chambre et l'accélératrice, nous avons fixé sur la chambre un cylindre métallique au même potentiel que cette dernière.

b) Les grilles. - Les grilles sont réalisées en fil de tungstène de $5 / 100 \mathrm{de} \mathrm{mm}$ de diamètre et ont une transparence de $95 \%$; ce qui donne un coefficient de transmission pour tout le spectromètre de $27 \%$. L'isolement des grilles est réalisé à l'aide de stéatites de $6 \mathrm{~mm}$ d'épaisseur.

c) Retardatrice. - La retardatrice est constituée par une grille de transparence plus faible, cette 


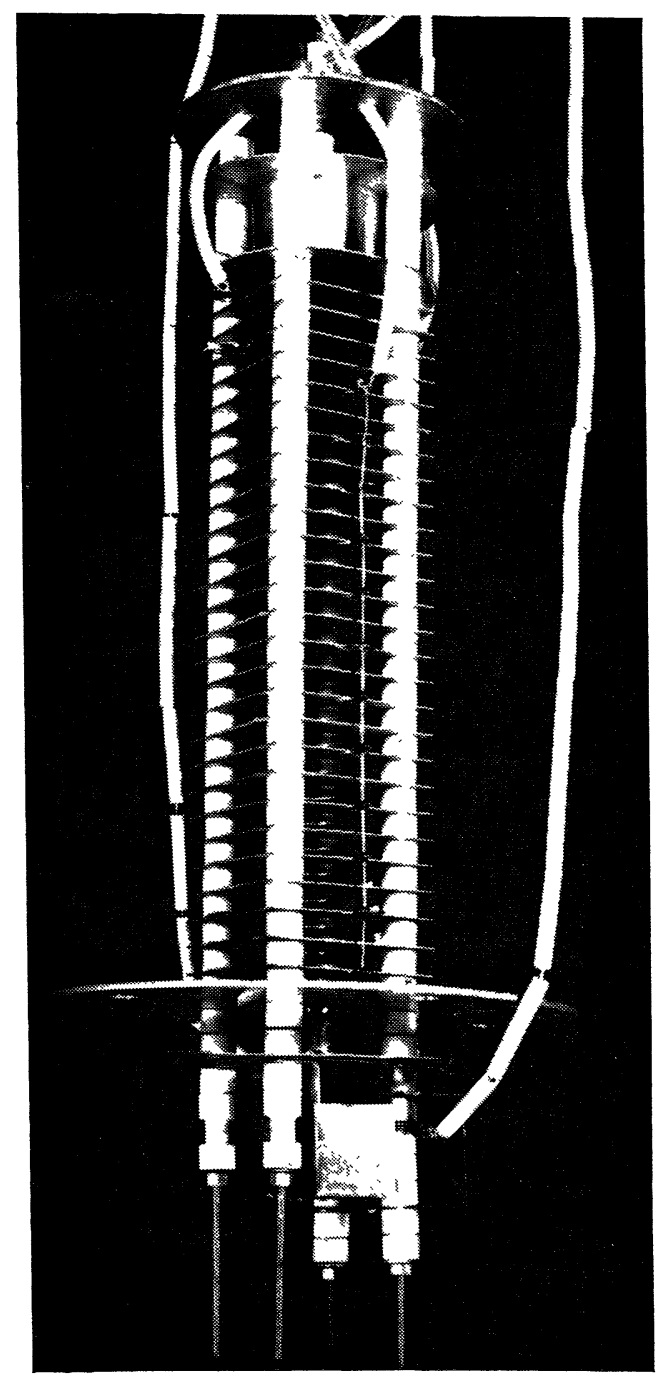

opacité relative entraînant une meilleure sélection des ions.

Deux grilles supplémentaires ont été disposées l'une à la masse en amont de la retardatrice, l'autre au potentiel de la cage de Faraday en aval de la retardatrice. Les effets perturbateurs dus à cette dernière sont ainsi supprimés.

d) Champ haute fréquence. - La tension haute fréquence est réglable de 0 à $5 \mathrm{~V}$ et la gamme de fréquence va de $250 \mathrm{kHz}$ à $50 \mathrm{MHz}$.

Nous avons utilisé un moteur tournant à faible vitesse pour réaliser un balayage lent et régulier en fréquence.

e) Mesure du courant ionique. - La mesure du courant obtenu sur le collecteur est faite à l'aide d'un électromètre dont la constante de temps pour les gammes les plus fréquemment utilisées lors de nos expériences est de $300 \mathrm{~ms}$.

L'électromètre est relié à un enregistreur qui nous a permis d'obtenir les résultats présentés ci-dessous.

Résultats. - Les auteurs des articles mentionnés ci-dessus donnent la courbe $\delta W_{\mathrm{m}}(\alpha) / \delta W_{\mathrm{m}}(\pi)$ tracée pour différentes valeurs de $N$. Nous avons pour notre part étudié expérimentalement la variation de $\delta W$ en fonction de $\alpha$.

Nos courbes expérimentales concordent bien avec les résultats théoriques. Nous avons choisi l'argon qui est bombardé par des électrons accélérés sous $90 \mathrm{~V}$. On observe bien (fig. 2) autour du pic le plus important correspondant à $\mathrm{A}+$ et $\alpha=\pi$ et autour du pic de $\mathrm{A}^{++}$une série d'ondulations.

Les ondulations pour les valeurs de $\alpha>\pi$ correspondant à $\mathrm{A}^{+}$étant masquées par celles de $\mathrm{A}^{++}$;

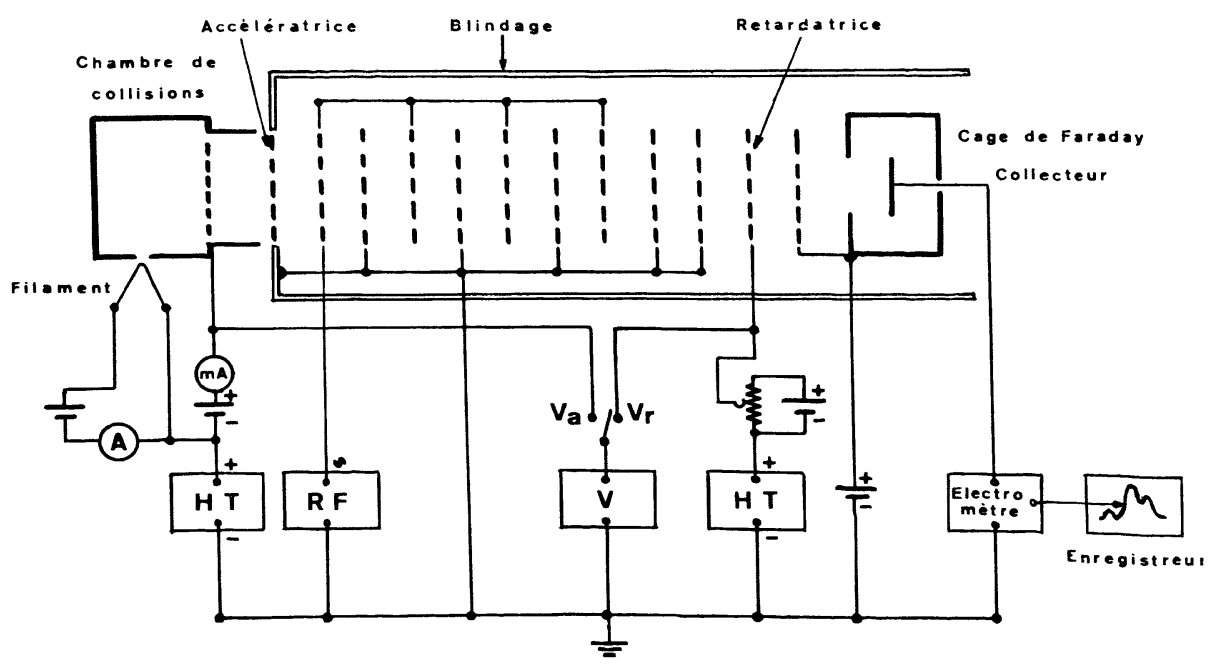

Fig. $1 b$. 


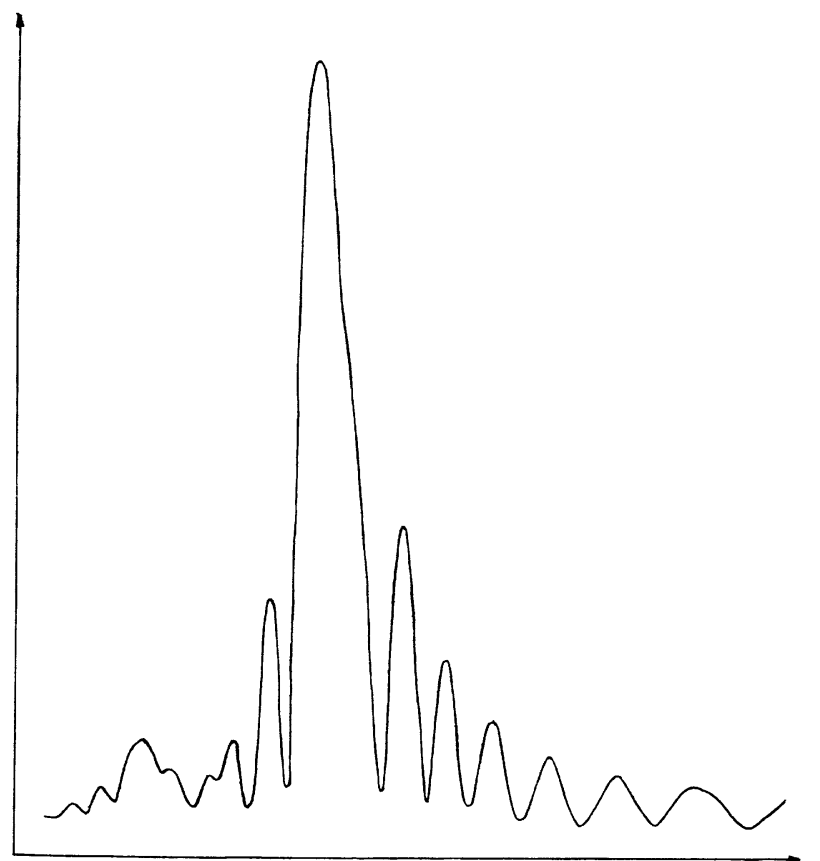

Fig. 2. - Ondulations autour des pics $\mathrm{A}^{+}$et $\mathrm{A}^{++}$. pour avoir un phénomène plus visible nous avons diminué la tension d'accélération des électrons a fin de ne créer que très peu d'argon deux fois ionisé. Le résultat est présenté sur la figure 3. Ces ondulations sont observées pour une tension d'accélération du faisceau ionique de $685 \mathrm{~V}$ et un potentiel $V_{\mathrm{r}}$ de $725 \mathrm{~V}$ sur la retardatrice. On constate que ce phénomène parasite diminue progressivemene et disparaît pour $V_{\mathrm{r}}=740 \mathrm{~V}$. On remarque également que notre résultat expérimental est en parfait accord avec les résultats théoriques de Redhead, en particulier, en ce qui concerne la dissymétrie. Les ondulations ont en effet une amplitude plus faible pour les valeurs de $\alpha>\pi$ que pour les valeurs de $\alpha<\pi$.

Etalonnage. - La relation reliant la masse de l'ion à sa fréquence de résonance est de la forme (1)

$$
M=K V_{\mathrm{a}} / f^{2}
$$

$V_{\text {a }}$ étant la tension d'accélération de l'ion.

L'étalonnage de l'appareil consiste donc en la détermination de la constante $K$. J'argon une fois ionisé étant obienu ( $f g .4 a$ ) pour la fréquence de 4,6 MHz, on en déduit

$$
K=1,24
$$

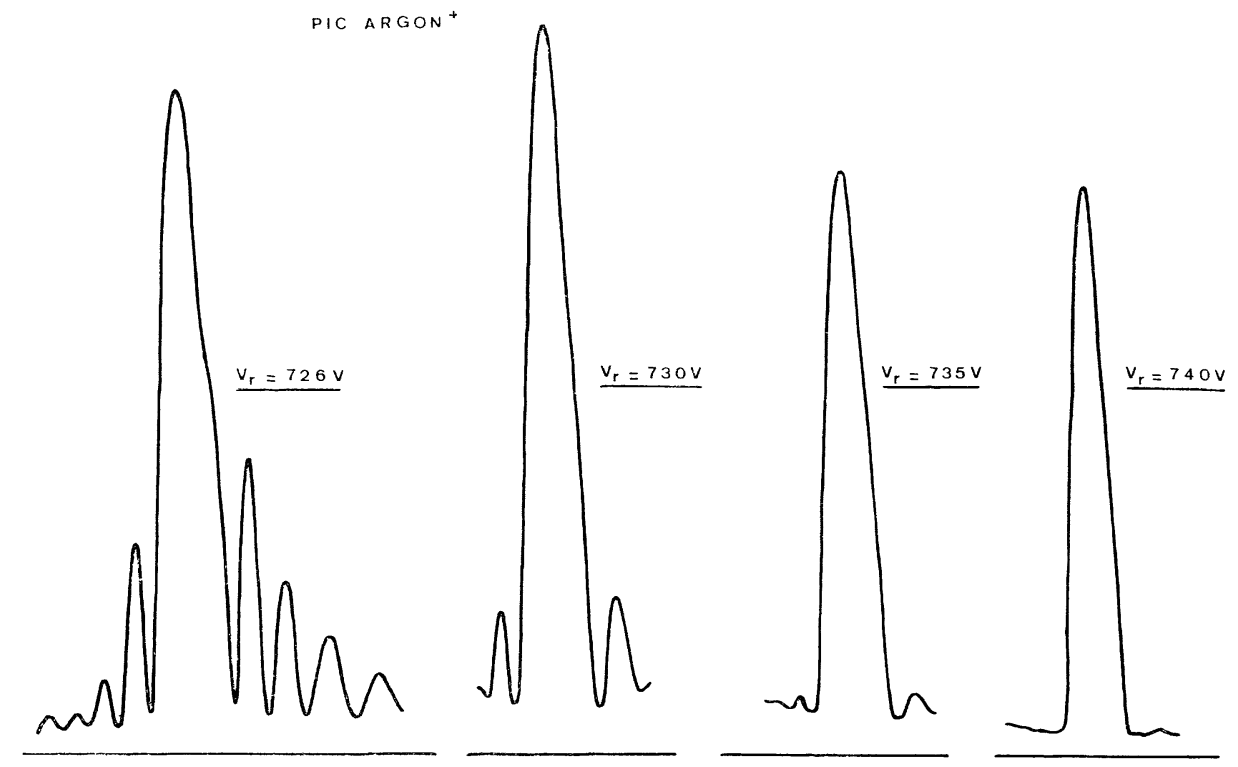

FIG. 3. - Action de la retardatrice sur les ondulations autour du pic $\mathrm{A}^{+}$.

si $V_{\mathrm{a}}$ est exprimé en volts et $f$ en Méga-Hertz.

Cette valeur a été également vérifiée à partir des ions $\mathrm{N}^{+}, \mathrm{N}_{2}^{+}, \mathrm{Kr}^{+}, \mathrm{O}+$ et $\mathrm{O}_{2}^{+}$.

Spectres de l'air. - Afin de comparer nos résultats avec ceux obtenus par Redhead [1] nous avons fait un spectre de l'air. Une partie de ce dernier est présenté sur la figure $4 b$. On remarquera que les rapports sont satisfaisants.
La figure 5 donne le spectre d'un mélange air vapeur d'eau. Des mesures plus précises dans ce domaine de fréquence nous ont permis de mettre en évidence un ion de masse 15 issu probablement des vapeurs d'huile en provenance du groupe de pompage lorsque le refroidissement du piège à l'azo ${ }^{ \pm}$ liquide n'était pas assuré.

Les résultats que nous présentons ci-dessus ont été obtenus avec le spectromètre à 24 étages. 


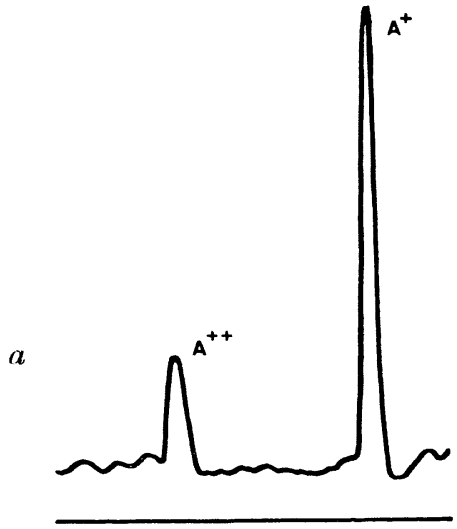

Fig. 4a. - Spectre de l'argon.

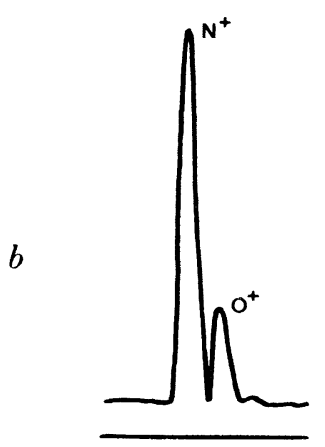

Fig. 4b. - Une partie du spectre de l'air.

Conclusion. - Le fonctionnement des spectromètres de masse que nous avons réalisés est parfaitement conforme aux prévisions théoriques dans le domaine de pressions où nous l'avons étudié $\left(10^{-5}\right.$ à $10^{-6}$ torr).

Le pouvoir de résolution du spectromètre de 24 étages est suffisant pour son utilisation en

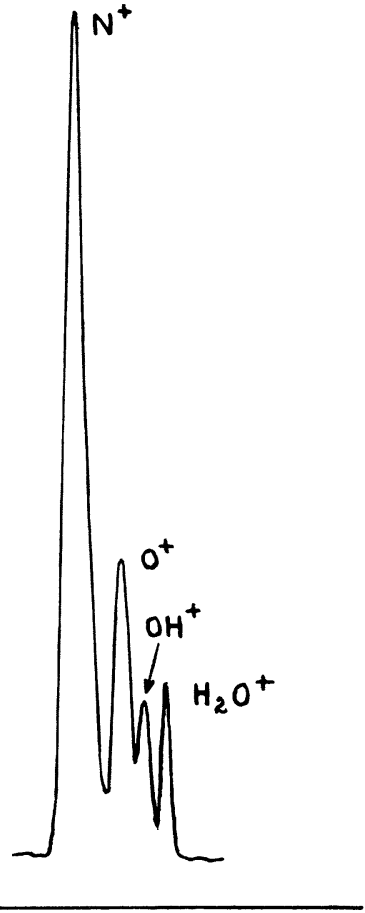

FIg. 5. - Spectre du mélange air-vapeur d'eau.

analyse; ceux de 8 et 16 étages peuvent servir à la production de faisceaux ioniques purs. Les spectromètres de ce type peuvent être utilisés aussi bien pour l'étude des ions négatifs que pour l'étude des ions positifs. En outre, ils sont peu encombrants, possèdent un coefficient de transmission élevé et ne nécessitent pas de champ magnétique. En raison de ces divers avantages, ils conviennent particulièrement aux montages d'études des sections efficaces d'ionisation.

Manuscrit reçu le 8 juillet 1966.

\section{BIBLIOGRAPHIE}

[1] Bennet (W. H.), Instruments, 1949, 22, 38.

[2] Bennet (W. H.), J. Appl. Physics, 1950, 21, 143.

[3] Redhead (P. A.), Canad. J. Phys., 1952, 30, 1.

[4] Redhead (P. A.) et Crowell (C. R.), J. Appl. Physics, 1953, 24, 331.
[5] Boyd (R. L. F.), Nature, 1950, 165, 142.

[6] Boyd (R. L. F.) et Morris (D.), Proc. Phys. Soc., 1955, 68, 1 . 